\title{
Outcomes of treatment of drug-susceptible tuberculosis at public sector primary healthcare clinics in Johannesburg, South Africa: A retrospective cohort study
}

\author{
E P Budgell, ${ }^{1}$ MSc; D Evans, ${ }^{1}$ PhD; K Schnippel,${ }^{2,3}$ MPA; P Ive,${ }^{3}$ MB BCh, FCP (SA), DTM\&H, Dip HIV Man (SA); L Long, ${ }^{1}$ MCom; \\ S Rosen, ${ }^{1,4} \mathrm{MPA}$ \\ ${ }^{1}$ Health Economics and Epidemiology Research Office, Department of Internal Medicine, Faculty of Health Sciences, University of the \\ Witwatersrand, Johannesburg, South Africa \\ ${ }^{2}$ Right to Care, Johannesburg, South Africa \\ ${ }^{3}$ Clinical HIV Research Unit, Department of Internal Medicine, Faculty of Health Sciences, University of the Witwatersrand, Johannesburg, \\ South Africa \\ ${ }^{4}$ Center for Global Health and Development, Boston University, Boston, USA
}

Corresponding author: S Rosen (sbrosen@bu.edu)

Background. Despite the large number of tuberculosis (TB) patients treated in South Africa (SA), there are few descriptions in the published literature of drug-susceptible TB patient characteristics, mode of diagnosis or treatment outcomes in routine public sector treatment programmes.

Objective. To enhance the evidence base for public sector TB treatment service delivery, we reported the characteristics of and outcomes for a retrospective cohort of adult TB patients at public sector clinics in the Johannesburg Metropolitan Municipality (JHB), SA.

Methods. We collected medical record data for a retrospective cohort of adult ( $\geq 18$ years) TB patients registered between 1 April 2011 and 31 March 2012 at three public sector clinics in JHB. Data were abstracted from National TB Programme clinic cards and the TB case registers routinely maintained at study sites. We report patient characteristics, mode of diagnosis, mode of treatment supervision, treatment characteristics, HIV status and treatment outcomes for this cohort.

Results. A total of 544 patients were enrolled in the cohort. Most (86\%) were new TB cases, $81 \%$ had pulmonary TB, $58 \%$ were smearpositive at treatment initiation and $71 \%$ were HIV co-infected. Among 495 patients with treatment outcomes reported, $80 \%(n=394)$ had successful outcomes, $11 \%(n=55)$ were lost to follow-up, $8 \%(n=40)$ died and $1 \%(n=6)$ failed treatment.

Conclusions. Primary healthcare clinics in JHB are achieving relatively high rates of success in treating drug-susceptible TB. Missing laboratory results were common, including follow-up smears, cultures and drug susceptibility tests, making it difficult to assess adherence to guidelines and leaving scope for substantial improvements in record-keeping at the clinics involved.

S Afr Med J 2016;106(10):1002-1009. DOI:10.7196/SAMJ.2016.v106i10.10745

In South Africa (SA), nearly one person in every hundred develops active tuberculosis (TB) each year (834 cases per 100000 people). Nearly two-thirds $(61 \%)$ of all notified TB cases are HIV-infected, and an estimated 96000 persons die from TB each year, 75\% of whom are HIV co-infected. ${ }^{[1]}$ Treatment for drug-susceptible tuberculosis (DS-TB) is provided at primary healthcare clinics (PHCs) using a standard regimen that requires daily medication for 6 - 8 months.

Despite the large number of patients treated in SA, there are few descriptions in the published literature of drug-susceptible patient characteristics, mode of diagnosis or treatment outcomes in routine public sector treatment programmes. What is evident from the published literature is that, even with clear national guidelines in place, ${ }^{[2,3]}$ practices and outcomes vary across the country. Differences in rates of treatment success are substantial, ranging from nearly $83 \%$ (Western Cape) to less than 58\% (Limpopo) at the provincial level, and from $90 \%$ (uThungulu, KwaZulu-Natal) to just $47 \%$ (Vhembe, Limpopo) at the district level. ${ }^{[4]}$ Methods for diagnosis vary, with nationally reported data suggesting that $40 \%$ of pulmonary TB cases are clinically diagnosed, ${ }^{[1]}$ although much higher rates may be observed among HIV-positive patients. ${ }^{[5]}$ Directly observed therapy (DOT) coverage rates and type of supervisor may also vary between facilities and by treatment phase. Data from Gauteng, KwaZulu-Natal and Mpumalanga provinces in 2009 indicated that while nearly all patients (97\%) received DOT support through the intensive phase of treatment, only $60 \%$ of patients received DOT support throughout the full treatment course. ${ }^{[6]}$

\section{Objective}

To contribute to the evidence base on public sector TB treatment service delivery, we constructed a retrospective cohort of adult TB patients treated at three public sector clinics in the city of Johannesburg. Using medical record data, we report patient characteristics, HIV status and TB history, diagnosis and laboratory information, treatment characteristics, DOT supervision and treatment outcomes for this cohort.

\section{Methods}

\section{Study sites}

The study was conducted at three PHCs in Region A of the Johannesburg Metropolitan Municipality (JHB) in Gauteng, SA. We selected a convenience sample of clinics with specific characteristics. Characteristics considered in the selection of study clinics included the volume of $\mathrm{TB}$ patients initiating $\mathrm{TB}$ treatment per month, the catchment population represented by the clinics and their location within the JHB region. At the time of the study, clinic 1 PHC, 
located in Ivory Park township, collected sputum samples from an average of 55 persons per month, with 6 patients per month testing acid-fast bacillus (AFB) smear microscopy-positive (District Health Information Software (DHIS), South Africa - unpublished data, 2011 - 2012). Clinic 2 PHC, located in Ebony Park township, tested a monthly average of 61 persons for TB, of whom 5 per month were smear microscopy-positive (DHIS - unpublished data, 2011 - 2012). Clinic 3 PHC, located in Diepsloot, a large informal settlement, tested an average of 82 persons per month for TB, of whom an average of
8 per month tested smear microscopy-positive (DHIS - unpublished data, 2011 - 2012). All three clinics followed national guidelines for drug-sensitive TB treatment in adults and adolescents in place at the time. ${ }^{[2]}$ Standards of care under these guidelines are detailed in Table 1.

\section{Study population and data}

In May 2013, a retrospective cohort was created from a census of adult ( $\geq 18$ years of age) TB patients registered in clinic case registers

Table 1. SA national guidelines for diagnosing and treating drug-sensitive TB during the study period (2011 - 2012)

Diagnosis

Registration and treatment initiation

Treatment regimen

Treatment monitoring

Treatment extensions

Resistance

Treatment outcomes

Co-infection with HIV
Diagnosis by AFB sputum smear microscopy. Two specimens sent to the National Health Laboratory Service (NHLS) laboratory associated with the clinic. Follow-up testing could be requested for patients who were AFB- but still TB symptomatic, including chest X-ray at nearby hospitals, or liquid culture and/or line probe assay at the provincial NHLS laboratory. Suspects with a history of prior TB treatment provided a third sputum sample for culture and DST. (Note: In 2011 SA began implementing a switch from AFB smear microscopy to GeneXpert MTB/RIF (Cepheid, USA) for the initial diagnosis of pulmonary TB, but the study period predated this roll-out.)

At the time of diagnosis, patients are either initiated on treatment and registered at the diagnosing facility, or initiated on treatment and immediately referred for registration and continuation of treatment at the most appropriate PHC based on the patient's home or work address. Those who are referred from TB hospitals to PHCs are provided with TB medication for 7 days and, if possible, are delivered to the clinic, collected by the clinic, or accompanied by a treatment supervisor or social worker. Where this is not possible, the hospital is expected to follow up directly with clinics to confirm whether the patient arrived. Patients are considered to have 'transferred in' if they were registered for TB treatment by another facility.

Standard 6-month chemotherapy regimen (regimen 1 ) of rifampicin $(R)$, isoniazid $(H)$, pyrazinamide $(Z)$ and ethambutol (E), which is administered daily as a fixed-dose combination tablet (RHZE 150, 75, 400, $275 \mathrm{mg}$ ) during an initial 2-month intensive phase, followed by a 4-month continuation phase of daily $\mathrm{R}$ and $\mathrm{H}$ (fixeddose tablet containing RH 150, $75 \mathrm{mg}$ or $300,150 \mathrm{mg}$, depending on weight). ${ }^{[2]}$ Guidelines recommended pyridoxine treatment for selected TB clients (i.e. if pregnant, diabetic, epileptic or abusing alcohol). ${ }^{[2]}$

Collection of a sputum specimen for smear microscopy at 2 months and 6 months. Patients with extrapulmonary TB or with a clinical diagnosis are assessed through clinical monitoring.

The intensive phase could be extended to 3 months in the absence of smear conversion, defined as remaining $\mathrm{AFB}+$ after 2 months of treatment. The continuation phase could be extended to 7 months in the event of severe or complicated disease such as miliary TB, meningitis, pericarditis, peritonitis, spinal or intestinal TB, or bilateral and extensive plural effusions. If a treatment interruption occurred lasting $<2$ months, treatment could also be extended by the number of days that the patient did not take treatment.

DST is performed for retreatment cases prior to starting treatment, for individuals who fail to smear-convert at the end of the intensive phase of treatment and for treatment failures. (Note: As of 2013, there was national coverage of GeneXpert MTB/RIF and therefore universal testing for rifampicin resistance. ${ }^{[3]}$ )

Patients considered cured were smear- or culture-positive at treatment initiation and smear- or culturenegative in the final month of treatment and on at least one occasion in the previous 30 days. Treatment completion refers to patients who completed treatment but did not meet the criteria to be classified as cured or as a treatment failure. Treatment failure refers to patients who were smear- or culture-positive at treatment initiation and remained smear- or culture-positive in the continuation phase, or who became smear- or culture-positive any time after treatment initiation, or whose DSTs indicated the presence of rifampicin resistance. Those who missed $>2$ consecutive months of treatment were considered to be lost to follow-up, while those who died included deaths from any reason during the course of treatment. Individuals who moved to another facility were recorded as transferred out; if the treatment outcome was unknown, the patient was assigned a missing outcome.

Patients co-infected with HIV were eligible for initiation on ART with a CD4 count below 350 cells/ $\mu \mathrm{L}$ or if there was evidence of MDR-TB or extensively drug-resistant TB, irrespective of CD4 count. Those who developed TB while on ART remained on ART throughout TB treatment. If eligible to begin ART, those who presented with TB before initiating ART were initiated on ART as soon as TB therapy was tolerated (typically within 2 - 4 weeks of initiating TB therapy, up to a maximum of 8 weeks). The standard firstline ART regimen consisted of tenofovir, lamivudine or emtricitabine, and efavirenz (which is generally preferred over nevirapine in TB patients). Co-trimoxazole preventive therapy was recommended and ideally initiated before starting ART. 
at the three sites between 1 April 2011 and 31 March 2012. Patients with missing outcomes were included in a descriptive analysis of the cohort but excluded from the final outcome analysis.

Data were extracted from National TB Programme (NTP) clinic cards and TB case registers routinely maintained at each study site. The data collected included age, sex, weight, treatment clinic, diagnosis method and date, registration and treatment start dates, transfer in, treatment history, type of TB, treatment regimens, laboratory results and dates, drug susceptibility testing (DST) results, type of treatment supervisor during both the intensive and continuation phases of treatment, HIV and antiretroviral therapy (ART) status at TB treatment initiation, CD4 count, and treatment outcomes and dates. The treatment supervisor was classified as formal supervision (including facility based, community caregiver or employer) and family- or self-administered treatment. Longitudinal information concerning HIV treatment, including ART regimens and laboratory results, was not available in the TB cards.

We attempted to locate missing data by checking $\mathrm{TB}$ case registers, referral slips and laboratory reports left in TB clinic cards. Individuals with blank DST results were assumed to have DS-TB; those with evidence of resistance were included in the baseline analysis of the cohort but later excluded from the final outcome assessment. Individuals who were registered for TB treatment on more than one occasion during the study period could be identified, as their TB cards were stapled together.

Smear microscopy status at treatment initiation could be determined only for pulmonary TB cases with: (i) a bacteriological diagnosis; or (ii) a clinical diagnosis if smear or culture results were also available. Scanty smears were categorised as smear-positive according to the SA NTP guidelines of $2009,{ }^{[2]}$ and smear conversion (i.e. conversion from smear-positive at treatment initiation to smearnegative) was estimated using laboratory results from the end of the intensive phase of treatment. Since the TB clinic cards did not distinguish between when sputum samples were collected and results obtained, it was not possible to discern whether patients with missing smear results had sputum samples collected.

\section{Outcomes and statistical analysis}

Treatment outcomes were defined according to the 2009 SA NTP guidelines (cured, treatment completed, treatment failed, died, lost to follow-up or transferred out), which were in use during the study period and recorded in the patient records (Table 1). ${ }^{[2]}$

We first summarised baseline demographic and clinical characteristics, mode of diagnosis, method of supervision, smear and culture conversion and treatment outcomes as proportions or medians with interquartile ranges (IQRs). We then investigated associations between baseline demographic and clinical characteristics and treatment outcomes. We modelled the risk of treatment success (a composite outcome of cured and completed treatment) and poor treatment outcome (a composite of died and failed) using modified Poisson regression with a robust error variance.

To obtain adjusted relative risk (RR) estimates, a priori confounders were identified and included in models for treatment success (adjusted for age, sex, treatment history and treatment supervisor) and poor treatment outcome (adjusted for age, sex and HIV status).

\section{Ethical considerations}

Approval for analysis was granted by the Human Research Ethics Committee (Medical) of the University of the Witwatersrand (clearance certificate number M130119) and the City of Johannesburg Health Department.

\section{Results}

\section{Baseline characteristics}

During the study period, 618 cases registered for TB treatment across the three facilities (Fig. 1). Seventy-four were not eligible for the study (63 minors, 11 missing files), leaving 544 patients included in the analysis of baseline characteristics. Fifty-six percent of patients were male $(306 / 544)$ and the median age was 35 years (IQR 29 - 41). Most (86\%) were new TB cases and most (81\%) had pulmonary TB. Seventy-one percent of cases were HIV co-infected (Table 2).

Among the pulmonary TB cases assessed for smear microscopy status ( $n=408), 58 \%$ were smear-positive at treatment initiation ( $61 \%$ among new cases and $44 \%$ among retreatment cases), and $16 \%$ were

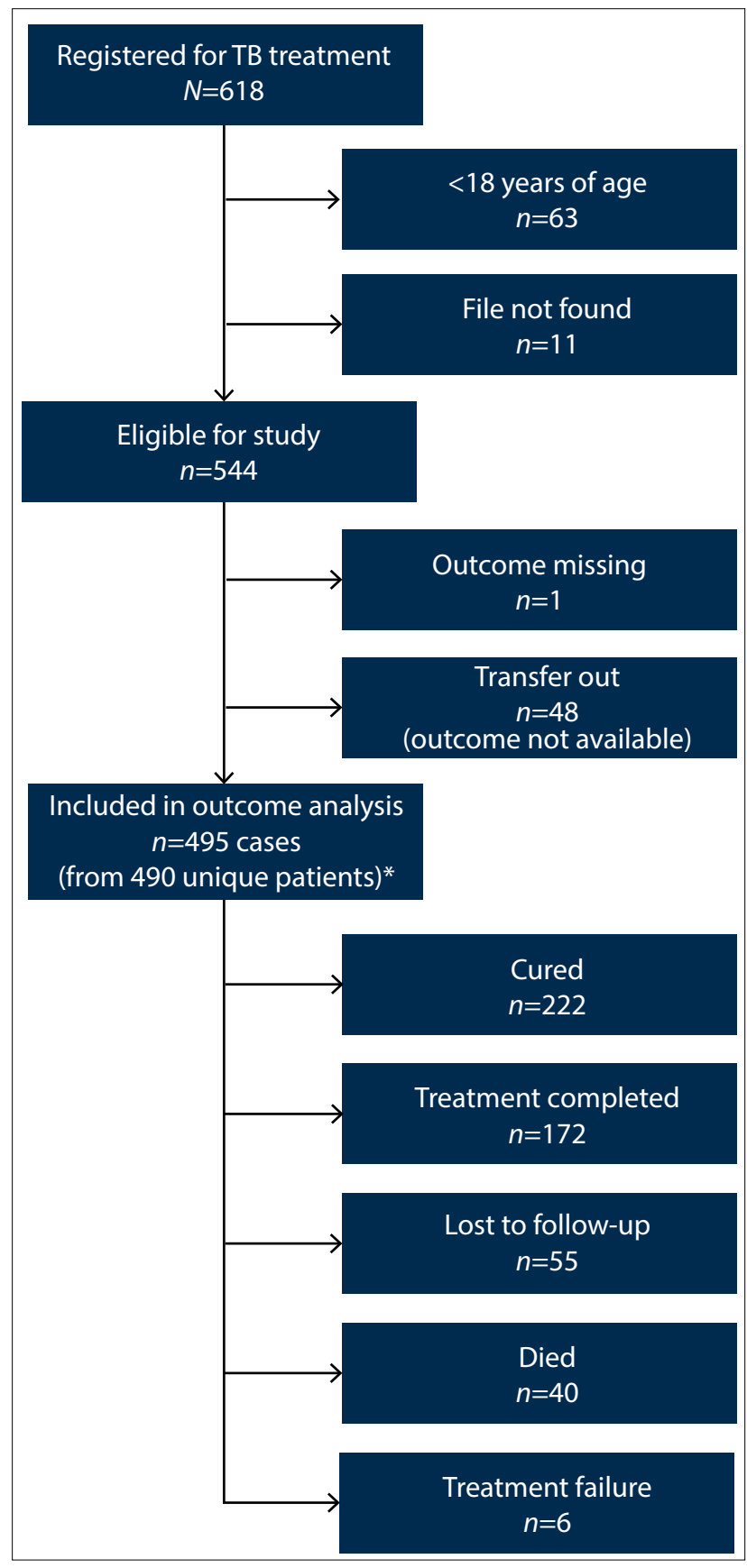

Fig. 1. Study enrolment and patient outcomes. ( ${ }^{*}$ Some patients were registered as two separate cases during the study period, once as a new case and once as a retreatment case.) 


\begin{tabular}{|c|c|c|}
\hline \multicolumn{2}{|l|}{ Characteristics } & \\
\hline Sex female, $n(\%)$ & $238(43.8)$ & Characteristics \\
\hline Age (yr), median (IQR) & $35(29-41)$ & Treatment supervisor (continuation phase), $n$ (\%) \\
\hline \multicolumn{2}{|l|}{ Weight (kg) } & Family- or self-administered \\
\hline Median (IQR) & $54.4(48.9-62.0)$ & $\begin{array}{l}\text { Formal supervision (clinic, community or } \\
\text { employer) }\end{array}$ \\
\hline Missing, $n(\%)$ & $35(6.4)$ & Supervisor unknown ${ }^{\varsigma}$ \\
\hline \multicolumn{2}{|l|}{ Patient category, $n(\%)$} & \multirow{2}{*}{ 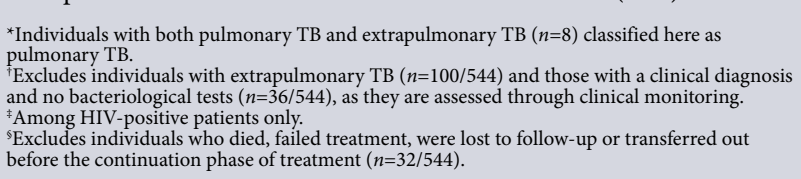 } \\
\hline Classification of disease, $n(\%)^{*}$ & $75(13.8)$ & \\
\hline Pulmonary TB & $443(81.4)$ & \\
\hline
\end{tabular}

$\begin{array}{ll}\text { Extrapulmonary TB } & 100(18.4) \\ \text { Missing } & 1(0.2)\end{array}$

Smear status, $n(\%)^{\dagger}$

Smear-positive

Smear-negative

Missing

Culture status, $n(\%)^{\dagger}$

Positive

Negative

Missing

HIV status

Positive

Negative

Missing

CD4 count $(\text { cells } / \mu \mathrm{L})^{\ddagger}$

Median (IQR)

Missing, $n(\%)$

ART, $n(\%)^{*}$

On ART

Not on ART

Missing

Method of diagnosis, $n$ (\%)

Smear microscopy

X-rays

Culture

GeneXpert MTB/RIF

Aspiration/biopsy

Cerebrospinal fluid

Ultrasound

Missing

Treatment supervisor (intensive phase), $n$ (\%)

Family- or self-administered

Formal supervision (clinic, community or employer)

Supervisor unknown
237 (58.1)

65 (15.9)

106 (26.0)

16 (3.9)

$1(0.2)$

391 (95.8)

388 (71.3)

101 (18.6)

55 (10.1)

$110.5(51.0$ - 236.0)

70 (18.0)

156 (40.2)

122 (31.4)

110 (28.4)

244 (44.9)

161 (29.6)

29 (5.3)

22 (4.0)

15 (2.8)

9 (1.7)

$1(0.2)$

63 (11.6)

278 (51.1)

$163(30.0)$

$103(18.9)$

Continued ... smear-negative; the remainder (26\%) did not indicate whether smear specimens were collected or tested.

\section{Diagnosis and treatment initiation}

Smear microscopy was the most common method of diagnosis (45\%), followed by X-rays (30\%), culture (5\%), GeneXpert MTB/ RIF (4\%), aspiration/biopsy (3\%), cerebrospinal fluid (2\%) and ultrasound $(<1 \%)$. Among HIV-positive cases, $34 \%$ of patients (130/388) were diagnosed with TB clinically. Some individuals with an unknown method of diagnosis (12\%) may have been diagnosed based on clinical symptoms alone, though it is unclear how many patients fell into this category, as symptom-based diagnoses were not recorded as such in the TB clinic cards.

Relatively few patients in the cohort initiated treatment at a study clinic. Nearly one-fifth $(101 / 544,19 \%)$ transferred in, primarily from within Gauteng (75/101, 75\%), followed by Limpopo (11/101, 11\%). Among those with no record of transferring in, over two-thirds of patients $(308 / 443,70 \%)$ initiated treatment prior to registering at a study clinic, suggesting that these individuals were first diagnosed at a non-study site, given a 1-week 'starter' supply of treatment medication, and referred for registration and continuation of treatment at a study site. Among these 308 individuals, the median time from treatment initiation to registration at a study clinic was 11 days (IQR 6 - 21).

\section{Guideline compliance}

When compliance with treatment guidelines was assessed, we found $96 \%$ of retreatment cases $(72 / 75)$ had a record of receiving streptomycin injections during the intensive phase, as per guidelines in place at the time of treatment. ${ }^{[2]}$ Nearly two-thirds $(63 \%, 341 / 544)$ of patients had a record of pyridoxine treatment and ART was recorded for $40 \%$ (156/388) of HIV-infected patients. For patients with HIV infection, guidelines also indicated concomitant co-trimoxazole prophylaxis, however this was only reported for a quarter $(28 \%, 153 / 388)$ of eligible patients. Other non-regimen drugs prescribed included vitamin $\mathrm{B}$ complex $(63 \%, 343 / 544)$, vitamin C $(26 \%, 143 / 544)$, ofloxacin $(<1 \%$, $2 / 544)$ and dapsone $(<1 \%, 2 / 544)$. Both of the patients prescribed ofloxacin had DST results available, with one indicating multidrugresistant (MDR) TB and the other indicating resistance to rifampicin. The quality of record-keeping for medications prescribed varied substantially by clinic, with clinics 1 and 3 recording other nonregimen drugs (e.g. vitamins) for $98 \%$ (125/127) and 88\% (219/250) of patients, respectively, compared with just $2 \%(4 / 167)$ at clinic 2 .

\section{Supervision}

Supervision type was reported for $81 \%$ of patients (441/544). Among those whose supervision type was reported, family or self-supervision 
was most common (intensive phase $63 \%$ of 441 , continuation phase $75 \%$ of 327 ), but there was a large range of family- or self-supervision rates across clinics (intensive phase: $75 \%$ for clinic $1,87 \%$ for clinic 2 , $43 \%$ for clinic 3; continuation phase: $96 \%$ for clinic $1,97 \%$ for clinic 2 , $53 \%$ for clinic 3). Among retreatment cases, 9\% (7/75) were reported as self-supervised and $24 \%(18 / 75)$ had no treatment supervisor listed during the intensive phase, although all had documentation of receiving anti-TB injections from the clinic; all retreatment cases were therefore assumed to be on facility-based supervision during the intensive phase. Supervision type was missing for 19\% (103/544) of cases in the intensive phase and in 36\% (185/512) of cases that reached the continuation phase of treatment.

\section{Treatment outcomes}

Because outcomes were not available for 49 patients (9\%), of whom 48 transferred out prior to reaching an outcome and 1 was missing an outcome, 495 patients were included in the final outcome analysis. Just over half $(52 \%, 124 / 237)$ of smear-positive cases achieved smear conversion at the end of the intensive phase of treatment, while $9 \%$ (22/237) did not smear-convert and the remaining 38\% (91/237) were missing the laboratory results needed to assess smear conversion. Nearly half of all cases (45\%) were cured and another $35 \%$ completed treatment, leading to an overall treatment success rate of $80 \%$. Eleven percent were lost to follow-up, $1 \%$ failed treatment, and $8 \%$ died (Table 3). The rate of treatment success was $80 \%$ among HIV co-infected patients,

Table 3. Treatment outcomes according to patient characteristics, among individuals with outcomes available $(N=495)$

\begin{tabular}{|c|c|c|c|c|c|c|c|}
\hline Characteristics & $\begin{array}{l}\text { Treatment } \\
\text { success, } \\
n(\%)\end{array}$ & $\begin{array}{l}\text { Cured, } \\
n(\%)\end{array}$ & $\begin{array}{l}\text { Treatment } \\
\text { completed, } \\
n(\%)\end{array}$ & $\begin{array}{l}\text { Lost to } \\
\text { follow-up, } \\
n(\%)\end{array}$ & $\begin{array}{l}\text { Treatment } \\
\text { failure, } \\
n(\%)\end{array}$ & Died, $n(\%)$ & Total, $n$ \\
\hline Total & $394(79.6)$ & $222(44.8)$ & $172(34.7)$ & $55(11.1)$ & $6(1.2)$ & $40(8.1)$ & 495 \\
\hline \multicolumn{8}{|l|}{ Sex } \\
\hline Male & $222(78.2)$ & $126(44.4)$ & $96(33.8)$ & $35(12.3)$ & $3(1.1)$ & $24(8.5)$ & 284 \\
\hline Female & $172(81.5)$ & $96(45.5)$ & $76(36.0)$ & $20(9.5)$ & $3(1.4)$ & $16(7.6)$ & 211 \\
\hline \multicolumn{8}{|l|}{ Age (yr) } \\
\hline $18-30$ & $122(81.9)$ & $69(46.3)$ & $53(35.6)$ & $20(13.4)$ & $2(1.3)$ & $5(3.4)$ & 149 \\
\hline$\geq 31-45$ & $206(80.2)$ & $111(43.2)$ & $95(37.0)$ & $26(10.1)$ & $1(0.4)$ & $24(9.3)$ & 257 \\
\hline$>45$ & $66(74.2)$ & $42(47.2)$ & $24(27.0)$ & $9(10.1)$ & $3(3.4)$ & $11(12.4)$ & 89 \\
\hline \multicolumn{8}{|l|}{ HIV status } \\
\hline Negative & $78(83.0)$ & $51(54.3)$ & $27(28.7)$ & $10(10.6)$ & $3(3.2)$ & $3(3.2)$ & 94 \\
\hline Positive, on ART & $110(80.3)$ & $50(36.5)$ & $60(43.8)$ & $13(9.5)$ & $0(0)$ & $14(10.2)$ & 137 \\
\hline Positive, not on ART & $93(80.2)$ & $49(42.2)$ & $44(37.9)$ & $12(10.3)$ & $0(0)$ & $11(9.5)$ & 116 \\
\hline Positive, ART unknown & $81(80.2)$ & $49(48.5)$ & $32(31.7)$ & $9(8.9)$ & $2(2.0)$ & $9(8.9)$ & 101 \\
\hline Unknown HIV status & $32(68.1)$ & $23(48.9)$ & $9(19.2)$ & $11(23.4)$ & $1(2.1)$ & $3(6.4)$ & 47 \\
\hline \multicolumn{8}{|l|}{ TB treatment history } \\
\hline New TB case & $339(78.7)$ & $195(45.2)$ & $144(33.4)$ & $51(11.8)$ & $6(1.4)$ & $35(8.1)$ & 431 \\
\hline Retreatment case & $55(85.9)$ & $27(42.2)$ & $28(43.8)$ & $4(6.3)$ & $0(0)$ & $5(7.8)$ & 64 \\
\hline \multicolumn{8}{|l|}{ Supervision type (intensive phase) } \\
\hline Supervised (formal) & $116(82.3)$ & $64(45.4)$ & $52(36.9)$ & $15(10.6)$ & $1(0.7)$ & $9(6.4)$ & 141 \\
\hline Family- or self-administered & $200(78.4)$ & $108(42.4)$ & $92(36.1)$ & $30(11.8)$ & $4(1.6)$ & $21(8.2)$ & 255 \\
\hline Unknown & $78(78.8)$ & $50(50.5)$ & $28(28.3)$ & $10(10.1)$ & $1(1.0)$ & $10(10.1)$ & 99 \\
\hline \multicolumn{8}{|c|}{ Supervision type (continuation phase) ${ }^{\dagger}$} \\
\hline Supervised (formal) & $65(84.4)$ & $38(49.4)$ & $27(35.1)$ & $7(9.1)$ & $1(1.3)$ & $4(5.2)$ & 77 \\
\hline Family- or self-administered & $211(90.6)$ & $115(49.4)$ & $96(41.2)$ & $11(4.7)$ & $3(1.3)$ & $8(3.4)$ & 233 \\
\hline Unknown & $118(70.7)$ & $69(41.3)$ & $49(29.3)$ & $31(18.6)$ & $2(1.2)$ & $16(9.6)$ & 167 \\
\hline \multicolumn{8}{|l|}{ Clinic } \\
\hline Clinic 1 & $97(78.9)$ & $73(59.3)$ & $24(19.5)$ & $13(10.6)$ & $3(2.4)$ & $10(8.1)$ & 123 \\
\hline Clinic 2 & $126(79.2)$ & $65(40.9)$ & $61(38.4)$ & $19(11.9)$ & $1(0.6)$ & $13(8.2)$ & 159 \\
\hline Clinic 3 & $171(80.3)$ & $84(39.4)$ & $87(40.8)$ & $23(10.8)$ & $2(0.9)$ & $17(8.0)$ & 213 \\
\hline \multicolumn{8}{|c|}{ Smear status at treatment initiation ${ }^{*}$} \\
\hline Smear-positive & $166(76.1)$ & $156(71.6)$ & $10(4.6)$ & $31(14.2)$ & $5(2.3)$ & $16(7.3)$ & 218 \\
\hline Smear-negative & $44(77.2)$ & $19(33.3)$ & $25(43.9)$ & $8(14.0)$ & $0(0)$ & $5(8.8)$ & 57 \\
\hline Unknown & $84(86.6)$ & $43(44.3)$ & $41(42.3)$ & $6(6.2)$ & $1(1.0)$ & $6(6.2)$ & 97 \\
\hline
\end{tabular}


$79 \%$ among new and relapse cases, and $83 \%$ among retreatment cases (excluding relapse). The cure rate was $71 \%$ (140/196) among new smear-positive cases and $73 \%(16 / 22)$ among retreatment smearpositive cases. Among patients who died, the median time to death was 78.5 days (IQR 55.0 - 124.5) from treatment initiation.

Comparing available laboratory results with outcomes recorded in patient records revealed that more than two-thirds (151/222, 68\%) of patients who were classified as cured were missing the laboratory tests needed to independently verify this outcome classification. For example, among the 219 cured pulmonary TB cases, one-fifth $(45 / 219,21 \%)$ lacked evidence of a positive bacteriological test at treatment initiation. Among cured pulmonary TB cases with a positive bacteriological test at baseline (174/219), more than half $(104 / 174,60 \%)$ were missing evidence of being smear- or culture-negative in the final month of treatment and on at least one occasion in the previous 30 days. Among cured extrapulmonary
TB cases $(n=3), 2$ patients had no laboratory results available to indicate cure. Of the 6 patients who were classified as having failed treatment, 4 were smear-positive at treatment initiation but lacked smear results from the continuation phase needed to confirm this outcome classification. Another 2 individuals appeared to have failed treatment but were classified as having completed treatment and died (i.e. treatment failure preceded date of death), respectively.

Adjusted models did not show any association between sex, HIV status, TB treatment history, or treatment supervisor and the risk of treatment success (Table 4 ). Older age ( $>45$ years) was suggestive of an increase in the risk of poor treatment outcomes compared with younger age (18 30 years) (adjusted RR 3.33, 95\% confidence interval (CI) 1.33 - 8.32).

\section{Resistance testing}

The SA NTP guidelines ${ }^{[2]}$ recommend DST for retreatment cases $(n=75)$ prior to starting treatment, for individuals who fail to smear-

Table 4. Risk of treatment success (cured or treatment completed) and poor treatment outcome (died or failed), according to patient characteristic $(N=495)$

\begin{tabular}{|c|c|c|c|c|}
\hline \multirow[b]{2}{*}{ Characteristics and exposure category } & \multicolumn{2}{|c|}{ Treatment success $(n=394)$} & \multicolumn{2}{|c|}{ Poor treatment outcome $(n=46)$} \\
\hline & $\begin{array}{l}\text { Crude RR } \\
(95 \% \mathrm{CI})\end{array}$ & Adjusted RR (95\% CI) & $\begin{array}{l}\text { Crude RR } \\
(95 \% \mathrm{CI})\end{array}$ & Adjusted RR (95\% CI) \\
\hline \multicolumn{5}{|l|}{ Age (yr) } \\
\hline $18-30$ & Ref & Ref & Ref & Ref \\
\hline $31-45$ & $0.98(0.89-1.08)$ & $1.02(0.81-1.30)$ & $2.07(0.92-4.67)$ & $1.96(0.83-.65)$ \\
\hline$>45$ & $0.91(0.78-1.05)$ & $0.91(0.67-1.23)$ & $3.35(1.40-7.98)$ & $3.33(1.33-8.32)$ \\
\hline \multicolumn{5}{|l|}{ Sex } \\
\hline Male & Ref & Ref & Ref & Ref \\
\hline Female & $1.04(0.95-1.14)$ & $1.00(0.82-1.24)$ & $0.95(0.54-1.66)$ & $1.03(0.57-1.89)$ \\
\hline \multicolumn{5}{|l|}{ HIV status } \\
\hline Negative & Ref & Ref & Ref & Ref \\
\hline Positive, on ART & $0.97(0.86-1.09)$ & $0.97(0.72-1.31)$ & $1.51(0.60-3.80)$ & $1.53(0.58-4.06)$ \\
\hline Positive, not on ART & $0.97(0.85-1.10)$ & $0.97(0.72-1.32)$ & $1.52(0.58-3.96)$ & $1.46(0.54-3.99)$ \\
\hline Positive, ART unknown & $0.97(0.85-1.10)$ & $0.99(0.72-1.36)$ & $1.68(0.65-4.38)$ & $1.62(0.59-4.43)$ \\
\hline Unknown HIV status & $0.82(0.66-1.02)$ & $0.86(0.57-1.30)$ & $1.33(0.40-4.50)$ & $1.28(0.36-4.54)$ \\
\hline \multicolumn{5}{|l|}{ TB treatment history } \\
\hline New patient & Ref & Ref & Ref & Not in model \\
\hline Retreatment & $1.09(0.98-1.22)$ & $1.11(0.76-1.64)$ & $0.82(0.34-2.00)$ & Not in model \\
\hline \multicolumn{5}{|l|}{ Clinic } \\
\hline Clinic 1 & Ref & Not in model & Ref & Not in model \\
\hline Clinic 2 & $1.00(0.89-1.13)$ & Not in model & $0.83(0.41-1.71)$ & Not in model \\
\hline Clinic 3 & $1.02(0.91-1.14)$ & Not in model & $0.84(0.43-1.65)$ & Not in model \\
\hline \multicolumn{5}{|l|}{$\begin{array}{l}\text { Treatment supervisor } \\
\text { (intensive phase) }\end{array}$} \\
\hline Supervised (formal) & Ref & Ref & Ref & Not in model \\
\hline Family- or self-administered & $0.95(0.86-1.05)$ & $0.90(0.62-1.30)$ & $1.38(0.68-2.79)$ & Not in model \\
\hline Supervisor unknown & $0.96(0.84-1.09)$ & $1.11(0.76-1.63)$ & $1.57(0.69-3.55)$ & Not in model \\
\hline \multicolumn{5}{|l|}{ Treatment supervisor (continuation phase)* } \\
\hline Supervised (formal) & Ref & Ref & Ref & Not in model \\
\hline Family- or self-administered & $1.07(0.97-1.19)$ & $1.19(0.82-1.72)$ & $0.73(0.26-2.03)$ & Not in model \\
\hline Supervisor unknown & $0.84(0.73-0.96)$ & $0.83(0.59-1.18)$ & $1.66(0.64-4.31)$ & Not in model \\
\hline
\end{tabular}


convert at the end of the intensive phase of treatment $(n=22)$, and for treatment failures $(n=6)$. Despite this, only $2 \%(2 / 97)$ of these patients had a record of DST being performed, including one with evidence of resistance to rifampicin and isoniazid (i.e. MDR-TB), and the other with evidence of resistance to rifampicin. An additional 4 patients had DST results but no clear indication for drug susceptibility testing; 2 showed blank drug resistance results, 1 had MDR-TB and 1 was resistant to both ethambutol and streptomycin. All individuals with evidence of drug resistance transferred out and are therefore not included in our analysis of treatment outcomes. Of the 6 patients with DST results, only 1 had evidence of drug sensitivity recorded.

\section{Discussion}

In this retrospective analysis of drug-sensitive $\mathrm{TB}$ treatment in Johannesburg, the three study clinics achieved reasonable rates of treatment success, averaging $80 \%$. When compared with nationally reported data, the study sites had higher rates of treatment success among previously treated cases (excluding relapse) (83\% v. 69\%) and similar rates of treatment success among new and relapse $(79 \%$ v. $78 \%$ ) and HIV-positive cases (80\% v. 76\%). ${ }^{[1]}$ Smear-positive cure rates were substantially lower (72\%) than the $85 \%$ target set in the 2009 SA NTP guidelines, ${ }^{[2]}$ and missing smear and culture results meant that the outcomes of more than two-thirds of patients reported as cured could not be independently verified.

We also note that only six patients had any record of DST taking place, despite 97 being eligible for DST. At best, this lack of documentation of laboratory results makes it difficult to assess adherence to guidelines; at worst, patients with a high potential for drug resistance are being missed, though it is unclear whether missing DST results necessarily implies testing was not done. An estimated $1.8 \%$ of new TB cases and $6.7 \%$ of retreatment cases have MDR-TB, ${ }^{[1]}$ suggesting that our cohort of 469 new cases and 75 retreatment cases may have had more than a dozen MDR-TB cases in need of secondline treatment, considerably more than the one individual with MDR-TB identified in this cohort. Our study therefore offers further justification for universal testing for resistance to rifampicin with the national adoption of Xpert MTB/RIF (Cepheid, USA) in 2011 as the first-line diagnostic test for $\mathrm{TB}$.

Although we found that three-quarters (409/544) of patients either transferred in or initiated treatment at a non-study site, we could not assess what fraction of patients may have failed to link to care after referral from other sites. Yet recent evidence from within SA suggests that referral outcomes may be unacceptably poor. Data from a district hospital in rural KwaZulu-Natal show that among HIV co-infected patients initiating TB treatment, $8 \%$ of those down-referred to PHCs failed to link to care and another $15 \%$ linked only after their supply of medication had run out, suggesting a gap in treatment. ${ }^{[5]}$ Other evidence from a large inner city hospital in Johannesburg shows that nearly a quarter (23\%) of TB patients failed to link to PHCs. Among the successful referrals, close to a third (30\%) took place after the patient's TB treatment supply expired, ${ }^{[7]}$ suggesting that there remains considerable room for strengthening linkage to TB care.

The study clinics had the same male/female ratio (1.3) seen in nationally reported data, ${ }^{[1]}$ although we did not observe gender differences across treatment outcomes, as has been documented in SA. ${ }^{[9]}$ We also found that $71 \%$ of patients were HIV co-infected, which is slightly higher than the national average of $61 \% .{ }^{[1]}$ While this rate is very high, the proportion of $\mathrm{TB}$ patients starting treatment with an unknown HIV status has dropped considerably in recent years (from $41 \%$ in 2009 to just $7 \%$ in 2014) and the proportion of HIVpositive TB patients on ART has increased dramatically (from 16\% in 2009 to $79 \%$ in 2014). ${ }^{[4]}$ Increasing CD4 counts ${ }^{[8]}$ and timely ART initiation are known to reduce mortality during TB therapy ${ }^{[9]}$ With SA guidelines prioritising the initiation of all HIV/TB co-infected patients (regardless of CD4 count or clinical staging), ${ }^{[3]}$ increases in ART coverage among TB patients are likely to continue, helping drive reductions in TB mortality.

Results from a systematic review have shown that in settings with high HIV prevalence and TB incidence, HIV infection, advancing immunosuppression, smear-negative disease and malnutrition are risk factors for TB mortality. ${ }^{[1]}$ Although we did not observe poorer treatment outcomes in HIV-positive patients, we did find that older age was associated with poor treatment outcomes, as documented elsewhere in SA. ${ }^{[8]}$ Older adults are more likely to experience comorbidities, drug-related adverse events, atypical forms of disease and extrapulmonary tuberculosis, which complicate diagnosis and treatment. ${ }^{[10]}$ Although studies of TB among the elderly are limited in SA, data from Soweto confirm the very high rates of extrapulmonary TB (50\%) and predisposing diseases (46\%) in TB patients aged $\geq 65$ years, ${ }^{[12]}$ suggesting that older adults may pose a growing challenge to TB control in the future. SA's HIV epidemic is also ageing, with the prevalence of HIV in people aged $\geq 50$ years expected to double in the next 30 years. ${ }^{[13]}$ With HIV co-infection being the greatest risk factor for developing active TB and TB-related deaths, ${ }^{[1]}$ the contribution of older HIV-positive patients to SA's TB burden is likely to increase.

Although it is not clear how effectively DOT was implemented in this study, the relatively high rate of treatment success among all study participants (with or without trained supervisors) suggests that trained supervision had little impact on this outcome compared with self-administered or family-supervised treatment. This interpretation is in line with a recent Cochrane review, ${ }^{[14]}$ which evaluated the results of 11 randomised controlled trials (5 662 participants) and concluded that there was little evidence that DOT has an impact on adherence during TB treatment. These findings suggest that self-administered TB treatment could potentially free up caregiver or healthcare worker time and clinic space for other health interventions.

\section{Study limitations}

This study has several limitations. Data were collected from TB registers and patient records available at the study sites, which varied in their completeness. Although we attempted to locate missing information, data could not always be found or verified, as was often true of laboratory results and the timing of HIV diagnosis. Clinic staff may have made errors in the transcription of treatment supervision type, or had different understandings of how supervision was defined. Since treatment supervision is captured at the start of each phase of $\mathrm{TB}$ treatment, any subsequent changes to supervision will have gone undocumented.

\section{Conclusions}

Based on the information recorded on the NTP clinic cards, the results of this analysis suggest that there is scope for substantial improvements in record keeping at these study sites, particularly for follow-up smears, cultures and DST results. Records of study visits, including the timing of scheduled visits $\mathrm{v}$. actual visits and drug dispensing, are often confined to study notes or recorded haphazardly elsewhere on the clinic cards, despite TB cards containing a dedicated space to record this information. These problems may be resolved through careful staff training and performance monitoring, but other issues stem from the design of TB clinic cards themselves. For example, NTP clinic cards do not provide dedicated space to record whether laboratory tests were ordered in the first place, and cards printed since 2011 do not record type of DOT treatment supervisor. Despite these limitations, the patient outcomes reported 
here compare well with national level data, while also highlighting ongoing challenges in meeting national targets for confirmed cure rates.

Funding source. This study was made possible by the generous support of the American people through the US Agency for International Development (USAID). Funding was provided by USAID under the terms of CA\# 674-A-12-00029 to the Health Economics and Epidemiology Research Office. The contents are the responsibility of the authors and do not necessarily reflect the views of USAID or the US government.

Conflict of interest. The authors have no conflicts of interest to disclose. Partial data were presented at the 4th SA TB Conference, Durban, SA, 10 13 June 2014, abstract no. 2637064.

Author contributions. EB, KS and SR conceived and designed the study. EB collected and analysed the data, and wrote the primary draft of the article. EB, DE, KS, PI, LL and SR contributed to the analysis and to writing the final manuscript.

Acknowledgements. The authors thank the patients and staff of the three clinics that participated in this study. We also thank the Gauteng and National departments of health for providing for the care of the patients. This study was made possible by the generous support of the Americans through Cooperative Agreement AID 674-A-12-00029 and Innovative Research on AIDS (INROADS) from USAID. The contents are the responsibility of the authors and do not necessarily reflect the views of USAID or the US government. EB, KS, DE, LL and SR were funded by this Cooperative Agreement. Any opinion, finding, and conclusion or recommendation expressed in this material is that of the author(s) and USAID does not accept any liability in this regard.

. World Health Organization. Global Tuberculosis Report 2015. Geneva: WHO, 2015. www.who.int/tb/ publications/global_report/en (accessed 2 March 2016).

National Department of Health, South Africa. National Tuberculosis Management Guidelines 2009. Pretoria: NDoH, 2009. http://familymedicine.ukzn.ac.za/Libraries/Guidelines_Protocols/TB_ Guidelines_2009.sflb.ashx (accessed 2 March 2016).

3. National Department of Health, South Africa. National Tuberculosis Management Guidelines 2014. Pretoria: NDoH, 2014. www.doh.gov.za/docs/hivAids/NationalTBManagementGuidelines.pdf (accessed 2 March 2016).

4. Massyn N, Peer N, Padarath A, Barron P, Day C. District Health Barometer 2014/15. Durban: Health Massyn N, Peer N, Padarath A, Barron P, Day C. District Health Barometer 2014/15. Durban: Health
Systems Trust, 2015. www.hst.org.za/publications/district-health-barometer-201415-1 (accessed 2 March 2016).

5. Jacobson KB, Moll AP, Friedland GH, Shenoi SV. Successful tuberculosis treatment outcomes among HIV/TB coinfected patients down-referred from a district hospital to primary health clinics in rural South Africa. PLoS One 2015;10(5):e0127024. DOI:10.1371/journal.pone.0127024

6. Ershova JV, Podewils LJ, Bronner LE, Stockwell HG, Dlamini SS, Mametja LD. Evaluation of adherence to national treatment guidelines among tuberculosis patients in three provinces of South Africa. S Afr Med J 2014;104(5):362-368. DOI:10.7196/SAMJ.7655

7. Voss de Lima Y, Evans D, Page-Shipp L, et al. Linkage to care and treatment for TB and HIV among people newly diagnosed with TB or HIV-associated TB at a large, inner city South African hospital. PLoS One 2013;8(1):e49140. DOI:10.1371/journal.pone.0049140

8. Pepper DJ, Schomaker M, Wilkinson RJ, de Azevedo V, Maartens G. Independent predictors of tuberculosis mortality in a high HIV prevalence setting: A retrospective cohort study. AIDS Res Ther 2015;12:35. DOI:10.1186/s12981-015-0076-5

9. Abdool Karim SS, Naidoo K, Grobler A, et al. Timing of initiation of antiretroviral drugs during Abdool Karim SS, Naidoo K, Grobler A, et al. Timing of initiation of antiretroviral
tuberculosis therapy. N Engl J Med 2010;362(8):697-706. DOI:10.1056/NEJMoa0905848

10. Waitt CJ, Squire SB. A systematic review of risk factors for death in adults during and after tuberculosis . Waitt C], Squire SB. A systematic review of risk factors for death in adults during
treatment. Int J Tuberc Lung Dis 2011;15(7):871-885. DOI:10.5588/ijtld.10.0352

11. Negin J, Abimbola S, Marais BJ. Tuberculosis among older adults - time to take notice. Int J Infect Dis 2015;32:135-137. DOI:10.1016/j.ijid.2014.11.018

2. Karstaedt AS, Bolhaar M. Tuberculosis in older adults in Soweto, South Africa. Int J Tuberc Lung Dis 2014;18(10):1220-1222. DOI:10.5588/ijtll.14.0210.

13. Hontelez JAC, Lurie MN, Newell M-L, et al. Ageing with HIV in South Africa. AIDS 2011;25(13):16651667. DOI:10.1097/QAD.0b013e32834982ea

4. Karumbi J, Garner P. Directly observed therapy for treating tuberculosis. Cochrane Database Syst Rev 2015;5:CD003343. DOI:10.1002/14651858.CD003343.pub4

Accepted 9 May 2016. 